\title{
The Design and Operation of a MEMS Differential Scanning Nanocalorimeter for High-Speed Heat Capacity Measurements of Ultrathin Films
}

\author{
Eric A. Olson, Mikhail Yu. Efremov, Ming Zhang, Zishu Zhang, and Leslie H. Allen
}

\begin{abstract}
A MEMS sensor has been developed for use as a calorimetric cell in an ultra-sensitive, thin-film, differential scanning calorimetric technique. The sensor contains a freestanding, thin $(30 \mathrm{~nm}$ to $1000 \mathrm{~nm})$, low-stress silicon nitride membrane with lateral dimensions of a few millimeters. This membrane, along with a thin $(50 \mathrm{~nm})$ metallization layer, forms a calorimetric cell with an exceptionally small addenda. This small addenda creates a very sensitive calorimetric cell, able to make heat capacity measurements of nanometer-thick metal and polymer films. The sensor fabrication and various design considerations are discussed in detail. The calorimetric technique and examples of applications are described.

[891]
\end{abstract}

Index Terms-Calorimetry, membranes, microelectromechanical systems, microsensors, nanoparticles, plastic films, thin films.

\section{INTRODUCTION}

H EAT energy is a fundamental characteristic of every phase transformation and chemical reaction. Calorimetry is the primary technique for investigations of the energetics of material transformations, and has been used as such for over a hundred years. The growing interest in investigating material at nanometer-sized scales, however, requires refinements to the technique and the development of new devices that are not currently commercially available.

The physical size of material can have a tremendous effect on its thermodynamic properties. Heat capacity measurements are useful in quantifying these changes in materials characteristics. Many properties of a thin film or a small particle can be considerably different than that of the same material in bulk form: electrical resistance increases [1]; indirect bandgap semiconductors emit visible light [2]; crystal structures even change [3], [4]. This is because surface and interfacial effects, negligible in bulk material, become dominant at small scales where the total fraction of atoms at the surface is significant.

For some time, it has been known that the melting temperature of small particles is lower than that of the bulk material [5],

Manuscript received July 28, 2002; revised December 19, 2002. This work was supported through National Science Foundation DMR 0108694 and American Chemical Society Petroleum Research Fund grant 37027-AC7. This paper was performed in part at the Cornell Nanofabrication Facility (a member of the National Nanofabrication Users Network), which is supported by the National Science Foundation under grant ECS-9731293, its users, Cornell University and industrial affiliates. Subject Editor C.-J. Kim.

The authors are with the Department of Materials Science and Engineering and the Coordinated Science Laboratory at the University of Illinois at UrbanaChampaign, Urbana, IL 61801 USA (e-mail: L-allen9@uiuc.edu).

Digital Object Identifier 10.1109/JMEMS.2003.811755
[6]. Recently, we have shown using a calorimetric technique that the heat of fusion decreases in a similar fashion [7]. We have also demonstrated the preference for certain "magic number" sizes in supported, self-assembling, indium particles on an amorphous surface [8], previously only seen in cluster beams [9], [10] and on epitaxial layers [11].

A traditional differential scanning calorimeter (DSC) can not be used to measure small samples on the order of nanograms. For conventional DSC, the energy required to heat the calorimetric cell itself (the addenda) would be large compared to either the energy required to heat the sample or the energy involved in the transformation of the sample. So, the signal of interest would be masked by the contribution from the addenda. Thus, techniques that are more sensitive are required. One of the obvious ways to increase the sensitivity is to make the calorimetric cell as small as possible, thus minimizing the effect from the addenda [7], [12]-[17].

The ever-developing field of microelectromechanical systems (MEMS) allows for the creation of new characterization probes for the study of material behavior at small scales. By integrating a membrane into the sensor and forming electrical circuitry as a thin metallization layer directly on the membrane, we create an ultra-low-profile calorimetric cell with a radically decreased addenda, and, consequently, with very high sensitivity [7]-[8].

Scanning calorimetry, in which the sample is heated steadily to an elevated temperature, is useful in that it can measure not only the sample's specific heat, but also the amount of heat (i.e. enthalpy) involved in a chemical or physical process. Although some size dependent effects can be observed with electron diffraction [6] or in a transmission electron microscope [18], MEMS-based scanning calorimetry can provide unique information about the energetics of the process. Other MEMS calorimetric techniques such as the membrane-based ac calorimetry used by Hellman [16], in which a small ac temperature variation is applied to the sample on the MEMS-fabricated membrane, can measure specific heat capacity with high precision, but are not typically used to determine an enthalpy of reaction or phase transformation.

The purpose of this article is to describe the principles of operation of our calorimetric technique, which we are using in our investigations of materials at the small scale, with a focus on the details of sensor fabrication. Also, material characteristics which can be measured (e.g. heat capacity of a sample as a function of temperature, latent heat of a transformation, melting temperature, glass transition temperature) along with selected 


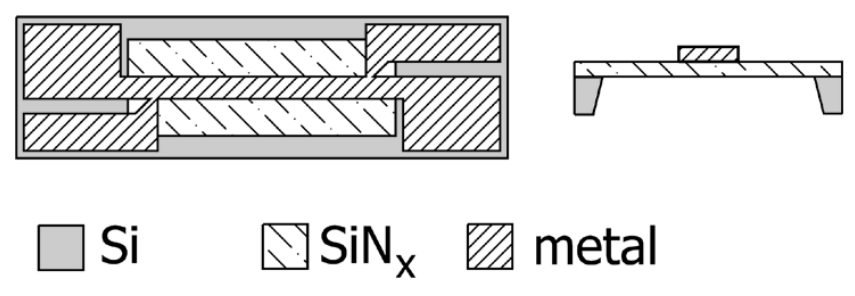

(a)

(b)

Fig. 1. Schematic of the calorimetric sensor. Plan view is shown in (a) and a cross section in (b). Metallization extends onto the Si frame in order that electrical connections can be made.

applications on ultrathin metal films and particles [7], [19], liquids [20], spin-coated films and isolated polymer crystals [21], [22] will be briefly discussed in order to demonstrate the utility of this method.

\section{MEMS-BASED THIN-FILM DSC: PRINCIPLES OF OPERATION}

The planar and cross-sectional configurations of a typical sensor for MEMS DSC are shown in Fig. 1. The sensor consists of a $50 \mathrm{~nm}$ thick amorphous silicon nitride $\left(\mathrm{SiN}_{\mathrm{x}}\right)$ membrane supported by a Si frame. The membrane window is $3 \mathrm{~mm} \times$ $7 \mathrm{~mm}$. A patterned metal strip is deposited on one side of the membrane. The strip is $50 \mathrm{~nm}$ thick and $0.5 \mathrm{~mm}$ wide. The strip is used as both a resistive heater and a resistive thermometer during the DSC measurement. In order to measure voltage across the working part of heater placed on the membrane, two voltage senses are formed on the membrane as a part of the patterned metallization layer. On the Si frame, the metallization pattern includes four contact pads, electrically connected with the strip and the senses, which serve as terminals for electrical measurements made on the membrane part of the sensor.

The region of the heater situated between the voltage senses and the part of membrane beneath it form a calorimetric cell. Samples can be loaded onto either side of the cell $\left(\mathrm{SiN}_{\mathrm{x}}\right.$ or metallization side), except for conductive materials, which are able to change the electrical properties of the heater and therefore should be placed only on $\mathrm{SiN}_{\mathrm{x}}$ side of the cell.

The calorimetric scan is initiated by applying a short (milliseconds) dc electrical current (tens of milliamperes) through the metal strip. The calorimetric cell heats by Joule heating, while the part of the strip placed on the Si frame remains cold due to the high heat capacity of the Si frame (in comparison with the $\mathrm{SiN}_{\mathrm{x}}$ membrane). The current $I$ through the sensor and the voltage $V$ across it (typically a few volts) are measured as functions of time $t$. The resistance of the sensing area of the heater is calculated as $R(t)=V(t) / I(t)$ and the electrical power generated in the calorimetric cell by Joule heating as $P(t)=I(t) V(t)$. The $R(t)$ function is used then to calculate the temperature $T(t)$ of the sensing part of the metal strip. To do this, the resistance of the sensor is calibrated against the temperature in a three-zone tube vacuum furnace.

To obtain a sensor with a stable electrical response, a two-step annealing procedure is performed before calibration. First, sensors are annealed in vacuum of $\sim 10^{-6} \mathrm{~Pa}$ at typically $450{ }^{\circ} \mathrm{C}$ for $8 \mathrm{~h}$. The temperature of annealing should be increased if calorimetric data above $350{ }^{\circ} \mathrm{C}$ to $400{ }^{\circ} \mathrm{C}$ are desired. However, high annealing temperatures can lead to an increase in the electrical resistance of sensors. Second, sensors are "burned-in" under the same vacuum conditions using a dc electrical pulse to heat the sensor to a temperature higher that needed for the experiment. Three to ten thousand pulses are used to burn in and stabilize each sensor. Calibrated sensors with $\mathrm{Au}, \mathrm{Ni}$ or Pt metallizations can be kept in air at least for several months without noticeable degradation of their electrical characteristics. Immediately before a calorimetric experiment, the burning-in process is repeated.

Under adiabatic conditions, the heat capacity $C_{P}$ of a calorimetric cell (bare or with a sample on it) can be calculated by

$$
P(t) d t=C_{P}(t) d T
$$

since all the heat will be consumed to increase the temperature of the cell. To achieve nearly adiabatic conditions, the heating rate for a sensor should be much faster than the cooling rate. In vacuum better than $10^{-2} \mathrm{~Pa}$, where convection is negligible, the main mechanisms of heat loss are lateral thermal conduction through the membrane and metallization layer and infrared emission. Typical cooling rates for temperatures of $100{ }^{\circ} \mathrm{C}$ to $300{ }^{\circ} \mathrm{C}$ are several $\mathrm{K} / \mathrm{ms}$, so heating rates of tens of $\mathrm{K} / \mathrm{ms}$ and higher are mandatory for adiabatic measurements.

To use the advantages of a differential measurement, two identical sensors are used: a sample sensor, containing the material being measured, and a reference sensor, with no added material. DC current pulses through the sensors are precisely synchronized. Five electrical measurements are made simultaneously in this differential scheme: currents through sample and reference sensors $I_{S}$ and $I_{R}$, respectively, voltages across sample and reference sensors $V_{S}$ and $V_{R}$, respectively, and the differential signal, the voltage $\Delta V$, which reflects the difference between sensors made by the presence of the sample on one of them. The principle circuitry in the differential measurement is shown in Fig. 2. The sampling rate for electrical measurements is typically $100 \mathrm{kHz}$, which corresponds to a temperature resolution of $0.3 \mathrm{~K}$ for a heating rate of $30 \mathrm{~K} / \mathrm{ms}$.

\section{MEMS SENSOR FABRICATION}

Sensor fabrication is performed on the Cornell Nanofabrication Facility. The calorimetric sensor was designed to be a small device easily produced in large numbers using standard MEMS microfabrication techniques with operation design rule limit of $10 \mu \mathrm{m}$ or larger. If desired, it can be treated as a one-use, disposable item. Up to 39 sensors per 3-inch wafer are made at a time. A schematic cross section of the wafer at several steps of the fabrication process can be seen in Fig. 3. Fabrication sequence is as follows:

1) Double-side polished, (100) oriented, silicon wafers are coated on both sides with amorphous, low residual stress silicon nitride $\left(\mathrm{SiN}_{\mathrm{x}}\right)$ using an LPCVD process. Double-side polished wafers are required for photolithography on both sides of the wafer. The thickness of the $\mathrm{SiN}_{\mathrm{x}}$ varies from about 30 to $1000 \mathrm{~nm}$, depending on the application. Thicker $\mathrm{SiN}_{\mathrm{x}}$ gives greater mechanical durability, but leads to larger heat losses. Optionally, a 


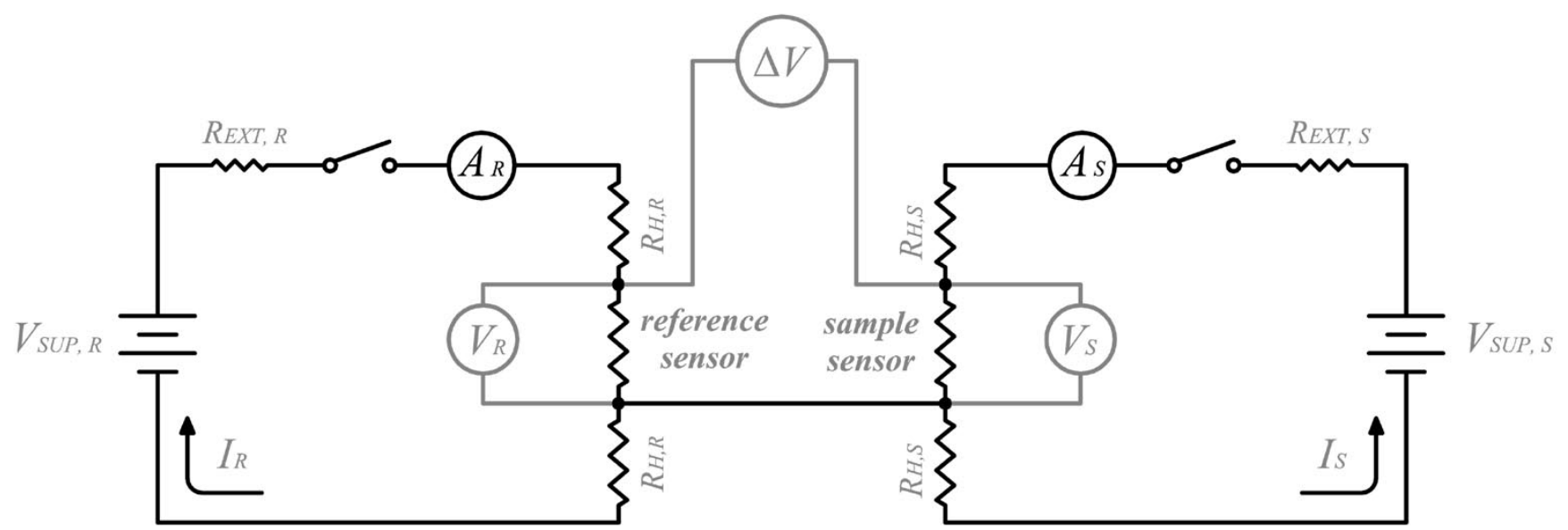

Fig. 2. Electrical schematic of the experimental apparatus. Two identical circuits are connected, and the differential voltage $\Delta V$ measured for maximum sensitivity. Both the reference and sample branches are composed of: a power supply $V_{\mathrm{SUP}}$, external resistance $R_{\mathrm{EXT}}$, and a calorimetric sensor. The voltage drop across the sensor $V$ is measured in each branch, as well as the current $I$ (by an ammeter $A$ ). Only the resistance of the heater between the voltage connections is measured. The small sections of the heater outside those connections are shown here as $R_{H}$.

thermally grown $\mathrm{SiO}_{2}$ layer between the wafer and $\mathrm{SiN}_{\mathrm{x}}$ can be made. The utility of such a layer will be discussed below.

2) On the back side of the wafer, a rectangular area of the $\mathrm{SiN}_{\mathrm{X}}$ is patterned using optical lithography. Shipley 1813 general purpose broadband ( $365 \mathrm{~nm}$ to $436 \mathrm{~nm}$ ) positive photoresist with MicroPrime P-20 primer are used for coating. A $5 \times$ g-line $(436 \mathrm{~nm})$ stepper (GCA-6300) is used for photolithography. After developing, the exposed part of the $\mathrm{SiN}_{\mathrm{x}}$ layer is etched away by reactive ion etching (RIE) using $\mathrm{CF}_{4}$. Since any damage to the unpatterned face of the wafer will create defects in the finished sensors, that face is protected during handling with a front-side coating of unexposed photoresist. Both front and rear side coatings are removed with acetone and 2-propanol after the RIE step.

3) The wafer is etched in a $35 \mathrm{wt} \% \mathrm{KOH}$ solution at $80{ }^{\circ} \mathrm{C}$ to remove $\mathrm{Si}$ exposed in the previous step. The etch rate for these conditions is $\sim 1 \mu \mathrm{m} / \mathrm{min}$. $\mathrm{KOH}$ has a high selectivity for silicon over silicon nitride. It also etches $\mathrm{Si}$ anisotropically, with a (100):(111) etch ratio of $~ 400: 1$ [23]. The resulting structure is a very thin $\mathrm{SiN}_{\mathrm{x}}$ membrane on the front side of wafer, supported by a Si frame. Wafers must be oriented vertically in the etchant, as vigorous bubbling can rupture the membranes. The end of the etching process is marked by the cessation of any bubbling action.

4) After washing in hot $\left(65^{\circ} \mathrm{C}\right)$ and then cold $\left(25^{\circ} \mathrm{C}\right)$ water, the wafer is then isotropically etched in a solution of $450 \mathrm{~mL} \mathrm{HNO}_{3}: 225 \mathrm{~mL} \mathrm{H} \mathrm{O}: 3 \mathrm{~mL} \mathrm{HF}$ for $5 \mathrm{~min}$. This removes any microscopic residual Si pyramids with (111) oriented faces. This etchant is not selective to $\mathrm{Si}$, and etches $\mathrm{SiN}_{\mathrm{X}}$ at $\sim 0.5$ to $1 \mathrm{~nm} / \mathrm{min}$. This etch step can be prolonged to thin the nitride membrane. Typically, this step reduces the $\operatorname{SiN}_{\mathrm{x}}$ thickness by $\sim 5 \mathrm{~nm}$. This factor should be taken into account when selecting the initial $\mathrm{SiN}_{\mathrm{x}}$ thickness. Since wafers have extremely fragile (a)

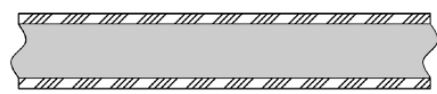

(b)

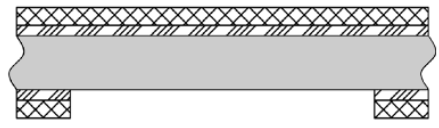

(c)

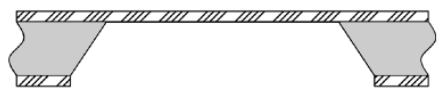

(d)

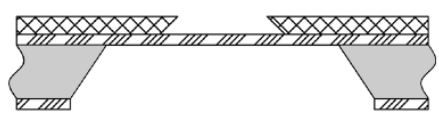

(e)

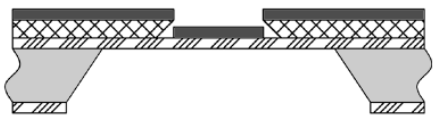

(f)
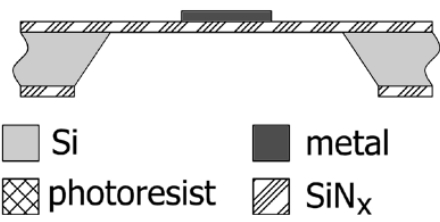

Fig. 3. Schematic cross section of the sensor at several points during fabrication. (a) LPCVD $\mathrm{SiN}_{\mathrm{x}}$ is deposited on both sides of a wafer. (b) Photoresist is added, patterned, and a section of the $\mathrm{SiN}_{\mathrm{x}}$ dry-etched away. (c) Exposed $\mathrm{Si}$ is etched in a $\mathrm{KOH}$ solution. (d) Second photoresist layer is spun on, exposed, and developed. Before developing, the tone of the exposure is reversed. (e) A $50 \mathrm{~nm}$ metal layer is deposited by thermal or electron beam evaporation. (f) The finished sensor.

nitride windows in this and subsequent steps, great care is required in the handling of wafers, especially during washing and drying. (Only gentle flows of solvents and dry air/nitrogen at small angles to the wafer surface should be used.)

5) A patterned metal layer is deposited on the front side of the wafer using the lift-off technique. Photoresist is applied on the front side of wafer using the same reagents as 
in step (2) and exposed by the same stepper. Membranes usually survive the regular spin-coating process with special chucks, but no vacuum should be used. A noncontact method of photolithography is mandatory at this step because direct contact would break the $\mathrm{SiN}_{\mathrm{x}}$ membranes. An ammonia diffusion process in a YES LP-III vacuum oven is used to reverse the tone of the patterned image and to generate an undercut profile for successful lift-off. After developing, the metal layer is deposited onto the wafer using thermal or electron beam evaporation. The thickness of this layer is typically $\sim 50 \mathrm{~nm}$. Deposition of a thin $(\sim 3 \mathrm{~nm})$ layer of Ti before the main metallization is necessary to promote adhesion of the following layer. Lift-off is performed using acetone as a solvent. To ensure high quality sensors, care should be taken to completely remove all photoresist residue.

6) Individual sensors are cleaved from the wafer manually. Narrow lines etched into the wafer in step 3) help the accuracy of the cleaving. To clean the surface and remove any residue, sensors are washed before use in sequential baths of acetone, 2-propanol, and deionized water.

\section{THE ElEMENTARy Model of the NANOCALORIMETER}

To get a basic understanding of the working characteristics of a calorimetric cell, the elementary model of the nanocalorimeter is described below. For the differential scheme shown in Fig. 2, and using the expression $P=I^{2} R$, (1) can be rewritten for both cells as

$$
\begin{aligned}
I_{R}^{2} R_{R}(t) & =C_{P}^{\text {cell }} \frac{d T_{R}}{d t} \text { and } \\
I_{S}^{2} R_{S}(t) & =\left(C_{P}^{\text {cell }}+C_{P}^{X}\right) \frac{d T_{S}}{d t}
\end{aligned}
$$

where indexes $S$ and $R$ denote sample and reference cell, $C_{P}^{\text {cell }}$ and $C_{P}^{X}$ are the heat capacities for the bare cell and the sample, respectively. Here, we assume that currents and heat capacities do not depend on time (or temperature). Assuming that both parts of the differential scheme (including sensors) are identical, that $C_{P}^{X} \ll C_{P}^{\text {cell }}$, that the left-hand sides of (2a) and (2b) are approximately equal, and by combining these equations, we can express the sample's heat capacity as

$$
C_{P}^{X}=C_{P}^{\text {cell }}\left(\frac{d T_{S}}{d t}\right)^{-1}\left(\frac{d T_{R}}{d t}-\frac{d T_{S}}{d t}\right) .
$$

Since the temperature is calculated from the resistance measurements, the final term in (3) can be found using the expressions for resistances

$$
\begin{aligned}
& R_{R}(t)=\left[\lambda+\lambda_{T}\left(T_{R}(t)-T_{0}\right)\right] \frac{l}{w h^{\mathrm{Met}}} \text { and } \\
& R_{S}(t)=\left[\lambda+\lambda_{T}\left(T_{S}(t)-T_{0}\right)\right] \frac{l}{w h^{\mathrm{Met}}}
\end{aligned}
$$

where $\lambda$ is the electrical resistivity of the metal layer at temperature $T_{0}$ and $\lambda_{T}$ is the temperature coefficient of resistance. The distance between voltage probes and the width and thickness of the heater are $l, w$ and $h^{\mathrm{Met}}$, respectively. Differentiating and combining (4a) and (4b), we get

$$
\frac{d T_{R}}{d t}-\frac{d T_{S}}{d t}=\frac{w h^{\mathrm{Met}}}{\lambda_{T} l}\left(\frac{d\left(R_{R}-R_{S}\right)}{d t}\right) .
$$

Noticing that $I_{R} R_{R}-I_{S} R_{S}=\Delta V$ and currents are approximately equal, we can rewrite (5) as

$$
\frac{d T_{R}}{d t}-\frac{d T_{S}}{d t}=\frac{w h^{\mathrm{Met}}}{I_{R} \lambda_{T} l} \frac{d \Delta V}{d t} .
$$

Expressing $I_{R}$ from (2a) and using $R_{R}\left(T_{0}\right)$ from (4a) to approximate resistance, we get

$$
I_{R}=\sqrt{\frac{C_{P}^{c e l l} w h^{M e t}}{\lambda l} \frac{d T_{R}}{d t}} .
$$

Substituting $I_{R}$ from (7) into (6) and using the result in (3), we get:

$$
C_{P}^{X}=\frac{\sqrt{C_{P}^{\text {cell }}}}{\lambda_{T} v^{\frac{3}{2}}} \sqrt{\frac{\lambda w h^{\mathrm{Met}}}{l}} \frac{d \Delta V}{d t}
$$

where $v$ denotes the heating rate of both cells (since they are similar). Finally, using specific heat capacities $c_{P}=C_{P} /(\rho h l w)$ (where $\rho$ is density and $h$ is thickness), and expressing the signal of interest $(d \Delta V / d t)$ explicitly, we obtain

$$
\frac{d \Delta V}{d t}=\frac{c_{P}^{X} \rho^{X} h^{X}}{\sqrt{c_{P}^{S i N} \rho^{S i N} h^{S i N}+c_{P}^{\mathrm{Met}} \rho^{\mathrm{Met}} h^{\mathrm{Met}}}} \frac{\lambda_{T} l}{\sqrt{\lambda h^{\mathrm{Met}}}} v^{\frac{3}{2}}
$$

where indexes $\mathrm{SiN}$ and Met denote the properties of silicon nitride and the metallization, respectively.

Sensitivity of the nanocalorimeter can be expressed in terms of the signal-to-noise ratio (SNR). For the $d \Delta V / d t$ signal there are two major types of random noise: instrumentation noise $e_{I}$ (noise of the measurement equipment) and thermal (Johnson) noise $e_{T}$ of resistors. Instrumentation noise does not depend on the characteristics of the sensors. If this type of noise is prevalent, the SNR has the same functional form (from (9)), as the $d \Delta V / d t$ signal does

$$
\mathrm{SNR}_{I} \propto \frac{c_{P}^{X} \rho^{X} h^{X}}{\sqrt{c_{P}^{\mathrm{SiN}} \rho^{\mathrm{SiN}} h^{\mathrm{SiN}}+c_{P}^{\mathrm{Met}} \rho^{\mathrm{Met}} h^{\mathrm{Met}}}} \frac{\lambda_{T} l}{\sqrt{\lambda h^{\mathrm{Met}}}} v^{\frac{3}{2}} .
$$

Thermal noise $e_{T} \propto \sqrt{R_{R}+R_{S}+4 R_{\text {probe }}+R_{\text {add }}}$, where $R_{\text {probe }}$ is resistance of the voltage probe, and $R_{\text {add }}$ consists of contact resistances and resistance of connecting wires. Typically, we can take into account only the resistances of the heaters. Using $R_{R}\left(T_{0}\right)$ from (4a) to approximate $R_{R}$ and $R_{S}$, we find

$$
e_{T} \propto \sqrt{\frac{\lambda l}{w h^{\mathrm{Met}}}}
$$

and SNR in the case of $e_{T}$ dominant has the form:

$$
\operatorname{SNR}_{T} \propto \frac{c_{P}^{X} \rho^{X} h^{X}}{\sqrt{c_{P}^{\mathrm{SiN}} \rho^{\mathrm{SiN}} h^{\mathrm{SiN}}+c_{P}^{\mathrm{Met}} \rho^{\mathrm{Met}} h^{\mathrm{Met}}}} \frac{\lambda_{T} \sqrt{l w}}{\lambda} v^{\frac{3}{2}} .
$$

Equations (10) and (12) will be used below in discussion of sensor design. 


\section{Sensor Design Considerations}

\section{A. Membrane Thickness}

The $\operatorname{SiN}_{\mathrm{X}}$ membrane contributes a significant part of the addenda. Nevertheless, due to the relatively weak dependence of SNR on the membrane thickness (see (10) and (12)), using membranes up to even $1 \mu \mathrm{m}$ thick does not critically disrupt the sensitivity of the method and can be useful, as in the case of massive samples.

The $\mathrm{SiN}_{\mathrm{x}}$ film will be the source of mechanical support for the heater strip once the fabrication of the sensor is complete. The thicker this layer is, the more durable the finished sensor will be. Some samples, for example, several hundred nanometers thick, spin-cast, glassy polymer films of polystyrene can cause stress high enough to destroy a typical sensor with a membrane $\sim 40 \mathrm{~nm}$ thick. In this case, increasing the $\mathrm{SiN}_{\mathrm{x}}$ thickness tenfold eliminates the problem.

If transmission electron microscopy (TEM) will be performed on a sensor, a thin membrane is essential for obtaining high-resolution images. Thick $\operatorname{SiN}_{\mathrm{x}}$ layers cause a great deal of background "noise" in the TEM. Thin $\mathrm{SiN}_{\mathrm{x}}$ layers can cause image shifting problems. A $30 \mathrm{~nm}$ thick membrane gives a good trade-off between mechanical strength and TEM performance.

Below $\sim 15 \mathrm{~nm}$ thick, the $\mathrm{SiN}_{\mathrm{x}}$ coverage appears to be incomplete and there is insufficient protection against the $\mathrm{KOH}$ solution used in step (3) of sensor fabrication, above. When very thin $\mathrm{SiN}_{\mathrm{X}}$ films were used, the entire wafer was etched, rather than regular and well-defined areas.

There is a special issue concerning the electrically insulating properties of the $\mathrm{SiN}_{\mathrm{x}}$ layer at elevated temperatures. During the calibration procedure, where the resistance of a sensor is measured under different temperatures in a massive thermostat placed in a vacuum furnace, the sensor frame under the contact pads (silicon covered by a $\mathrm{SiN}_{\mathrm{X}}$ layer) becomes conductive and shunts the heater. In some cases, this shunting can be detectable even at temperatures near $200{ }^{\circ} \mathrm{C}$. It should be noted that this effect is observed only during the calibration and not during the calorimetric measurements because, during the DSC scan, the Si frame remains cold and provides no significant conductivity.

It should also be noted also that no noticeable shunting of the heater by a conductive sample on the membrane was observed during DSC scans up to $\sim 500^{\circ} \mathrm{C}$, which means the $\mathrm{SiN}_{\mathrm{x}}$ membrane itself is not conductive at these temperatures. In addition, the shunting is not observed when the substrate beneath the $\mathrm{SiN}_{\mathrm{x}}$ layer is completely nonconductive (i.e., if an $\mathrm{SiO}_{2}$ wafer is used instead of $\mathrm{Si}$ to make model sensors without membrane). This means shunting is not caused by surface effects or lateral conductivity of the supported $\mathrm{SiN}_{\mathrm{x}}$ layer.

Despite the unknown cause of the shunting effect, it is possible to significantly reduce the effect by increasing the thickness of insulation layer between metallization and $\mathrm{Si}$ substrate. This can be done simply by increasing the thickness of the $\mathrm{SiN}_{\mathrm{x}}$ layer. For example, a $400 \mathrm{~nm}$ thick $\mathrm{SiN}_{\mathrm{x}}$ layer beneath a Pt metallization effectively eliminates shunting up to $360{ }^{\circ} \mathrm{C}$. This method, however, requires a substantial increase of the membrane thickness, because methods that offer selective $\mathrm{SiN}_{\mathrm{x}}$ etching only the on membrane part (such as
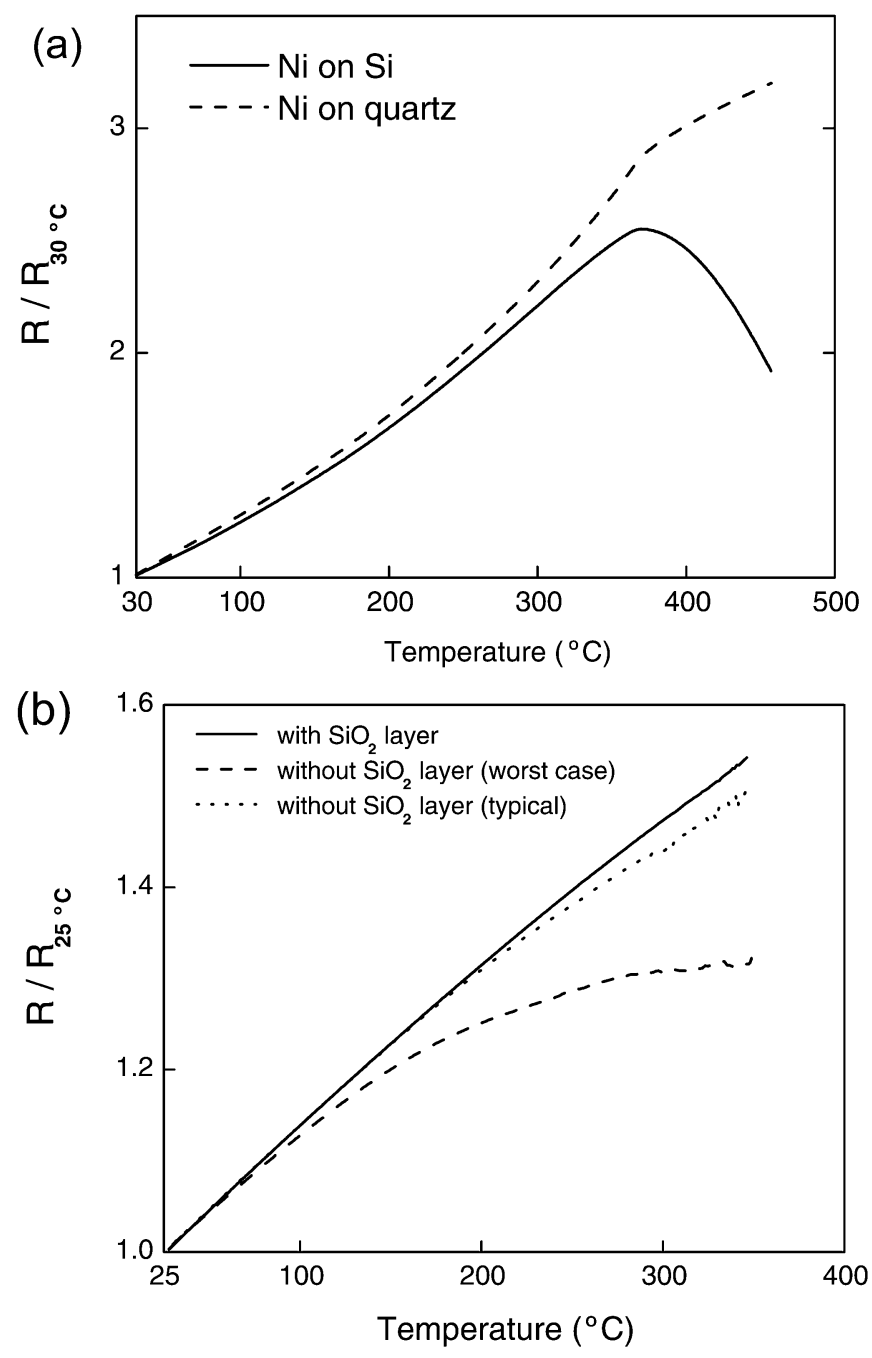

Fig. 4. Effect of Si frame resistance on calibration. Two Ni sensors are shown in (a), one $\mathrm{Ni}$ on $\mathrm{SiN}_{\mathrm{x}}$ on a $\mathrm{Si}$ frame (solid line), the other $\mathrm{Ni}$ on $\mathrm{SiN}_{\mathrm{x}}$ on a quartz wafer (dashed line). Ni undergoes a magnetic transition at $358^{\circ} \mathrm{C}$ [35], and the $R(T)$ curve changes slope. The drastic downturn in the Ni-on-Si case is due to decreasing contact resistance between the contact pin and the $\mathrm{Si}$ wafer. Part (b) shows the effect of adding a $100 \mathrm{~nm} \mathrm{SiO}$ layer between the $\mathrm{Si}$ and the $\mathrm{SiN}_{\mathrm{x}}$ film. Both worst-case and typical curves for sensors without the $\mathrm{SiO}_{2}$ layer are shown. The metallization used in (b) is Pt.

isotropic etching and RIE) do not provide fine enough control of etch depth at typical membrane thicknesses.

Another method uses the formation of an additional insulating layer, chemically different from $\mathrm{SiN}_{\mathrm{x}}$, between the $\mathrm{SiN}_{\mathrm{x}}$ layer and the Si substrate. Such an additional layer can be removed selectively from the membrane part of the sensor. Preliminary tests using a thermally grown $\mathrm{SiO}_{2}$ layer between the $\mathrm{Si}$ and $\mathrm{SiN}_{\mathrm{x}}$ are encouraging. The example shown in Fig. 4 incorporates a $100 \mathrm{~nm}$ thick $\mathrm{SiO}_{2}$ layer. By the end of fabrication, the $\mathrm{SiO}_{2}$ layer exists only in the frame, as it is etched in the $\mathrm{KOH}$ etch step. If the oxide layer is too thin, the effectiveness of isolation is reduced. If the oxide layer is too thick, the resulting $\mathrm{SiN}_{\mathrm{x}}$ membrane becomes stressed. When fabricated with $200 \mathrm{~nm}$ and $400 \mathrm{~nm}$ oxide layers, membranes buckled and were no longer flat, which caused problems in the metallization lithography step. 
TABLE I

The Materials That Make Up The Calorimeter and Their Bulk Physical and Electrical Properties at 298 K

\begin{tabular}{|c|c|c|c|c|c|c|}
\hline Material & $\begin{array}{c}\rho \\
\left(\mathrm{g} \cdot \mathrm{cm}^{-3}\right)\end{array}$ & $\begin{array}{c}c_{P} \\
\left(\mathrm{~J} \cdot \mathrm{g}^{-1} \cdot \mathrm{K}^{-1}\right)\end{array}$ & $\begin{array}{c}\mathrm{k} \\
\left(\mathrm{W} \cdot \mathrm{cm}^{-1} \cdot \mathrm{K}\right)\end{array}$ & $\begin{array}{c}\lambda \\
(\mu \Omega \cdot \mathrm{cm})\end{array}$ & $\begin{array}{c}\lambda_{T}^{\mathrm{a}} \\
\left(\times 10^{-3} \mathrm{~K}^{-1}\right)\end{array}$ & Ref. \\
\hline $\mathrm{Ag}$ & 10.5 & 0.235 & 4.29 & 1.617 & 4.10 & [29], [30] \\
\hline $\mathrm{Al}$ & 2.7 & 0.904 & 2.37 & 2.709 & 4.26 & [29], [30] \\
\hline $\mathrm{Au}$ & 19.3 & 0.129 & 3.17 & 2.255 & 3.98 & {$[29],[30]$} \\
\hline Co & 8.86 & 0.421 & 1.00 & $6.5^{\mathrm{b}}$ & 6.6 & {$[29],[30]$} \\
\hline $\mathrm{Ni}$ & 8.90 & 0.445 & 0.907 & 7.12 & 6.7 & [29], [30] \\
\hline $\mathrm{Pd}$ & 12.0 & 0.244 & 0.718 & 10.73 & 3.68 & [29], [30] \\
\hline $\mathrm{Pt}$ & 21.5 & 0.133 & 0.716 & 10.7 & 3.92 & {$[29],[30]$} \\
\hline $\mathrm{Ti}$ & 4.51 & 0.522 & 0.219 & $44^{\mathrm{b}}$ & 3.3 & [29], [30] \\
\hline $\mathrm{SiN}_{\mathrm{x}}$ & 3.0 & 0.7 & 0.032 & $\ldots$ & $\ldots$ & [31] \\
\hline
\end{tabular}

\section{B. Metal Heater: Material Selection}

The metals we have used for the heater strip include Ag, $\mathrm{Al}, \mathrm{Au}, \mathrm{Co}, \mathrm{Ni}, \mathrm{Pd}$, and Pt. This is not an exhaustive list, however, and other materials may be suitable depending on the application.

From the point of view of sensitivity, a good material for metallization should have a high temperature coefficient of resistance $\lambda_{T}$ and low specific resistance $\lambda$ [see (10) and (12)]. The bulk $\lambda$ and $\lambda_{T}$ for several heater materials, as well as other properties, are listed in Table I.

The effective $\lambda_{T}$ for our sensors tends to be $\sim 50 \%$ of the bulk. This lower than ideal response could be due to thin-film effects [24], the polycrystalline nature of the film, and/or the effect from the metal alloying with the Ti adhesion layer.

Decreasing of $\lambda$ alone is not an effective way to improve the device characteristics. It leads to instrumental noise being dominant in the differential signal, where the SNR is not very sensitive to $\lambda$ [see (10)]. Additionally, maintaining the same heating rates for highly conductive heaters requires [according to (7)] an increase in the magnitude of the current pulse through the sensor, which causes unfavorable transient processes at the front and the end of the pulse to become more pronounced. However, decreasing the heater resistance strongly reduces the shunting effect during the sensor calibration procedure, described above. This effect is obvious, since shunting is proportional to the ratio of the shunt and shunted resistances.

For optimal results, the electrical resistivity and heat capacity of the material used for the heater strip should have a linear thermal response in the temperature range of interest. Any nonlinearities in the $R(T)$ and $C_{P}(T)$ dependencies of the heater will be strongly amplified in the differential signal.

It should be noted that most sensors have small feature in their $C_{P}(T)$ response between $250{ }^{\circ} \mathrm{C}$ and $300{ }^{\circ} \mathrm{C}$, which causes noticeable artifacts within the differential signal at this temperature range. This spurious signal varies with the metal used for the heater and is practically negligible for $\mathrm{Au}$. The stability of electrical properties of the heater during multiple DSC scans should be high enough to maintain accurate and reproducible results. For instance, $\mathrm{Pt}$ is the preferred material over $\mathrm{Au}$ and $\mathrm{Ni}$ if stability is critical for given applications.

Ferromagnetic materials such as $\mathrm{Ni}$ and Co have a high $\lambda_{T}$, and so have a sensitivity advantage. There are some drawbacks, however. The shape of the $T(R)$ dependence tends to be less linear than for a nonferromagnetic metal such as Au or Pt. Both the $T(R)$ and $C_{P}(T)$ curves deviate strongly from linear at the Curie point for a ferromagnetic material. Such metals also make electron microscopy more difficult, as the magnetic nature of the material interferes with the electron beam, making imaging small features extremely difficult. Light metals, like Al, are particularly good for TEM studies, as it is possible to image samples directly through the heater.

Should Rutherford backscattering spectrometry (RBS) be desired, it is important to select a heater material that will not interfere with the RBS signal from the sample being investigated. This requires that the atomic number of the material of the heater should be significantly different from those of the sample's constituent elements.

Some applications require reduced chemical activity of the metallization layer. For example, to prevent the growth of selfassembled monolayers of alkanethiols directly on the heater, Al is preferred over $\mathrm{Ag}, \mathrm{Au}, \mathrm{Pd}$, or Pt. [25].

As mentioned above, the Ti adhesion layer can cause problems in very sensitive measurements. Experiments with the other frequently used material for an adhesive layer, $\mathrm{Cr}$, show no obvious improvement. Some active metals, like Al, can stick to $\mathrm{SiN}_{\mathrm{x}}$ without any adhesive underlayer. Preliminary tests of sensors with pure Al metallization show promising results in terms of stability and the reduction of spurious signals.

\section{Metal Heater: Thickness}

Like the $\mathrm{SiN}_{\mathrm{x}}$ membrane, the heater contributes to the significant part of the addenda, and sensitivity can be enhanced by reducing of the heater thickness $h^{\text {Met }}$ [see (10) and (12)]. This would also improve TEM micrographs of sample on the heater part of sensor. However, making the heater thinner leads to domination of thermal noise in the signal, where SNR is not very sensitive to $h^{\text {Met }}$ [see (12)]. Additionally, reducing $h^{\text {Met }}$ has the detrimental thin-film effects of 1) decreasing $\left.\lambda_{T}, 2\right)$ increasing the likelihood that the metal strip itself is a discontinuous film, and 3) magnifying electromigration effects [26]. Increasing $h^{\text {Met }}$ will lead to a decrease in the severity of shunting effect during calibration and, at the same time, to an increase in the transient processes during measurement, as was described in the previous section for a decrease in $\lambda$. Thicker metal film makes ellipsometric measurements of sample thickness on the 


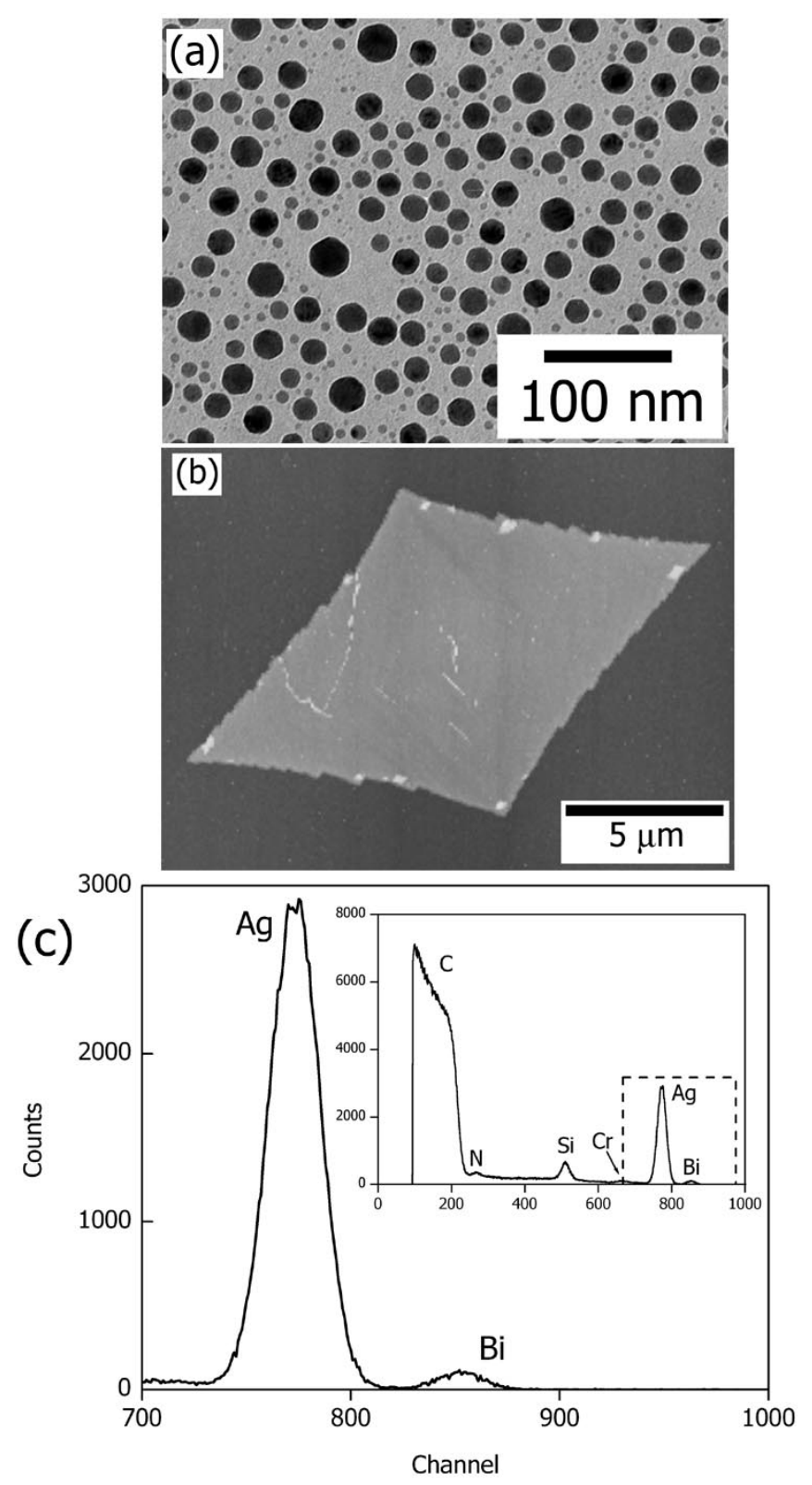

Fig. 5. Various microanalysis techniques used on calorimetric sensors. The micrograph in (a) is a plan view TEM image of $3 \mathrm{~nm}$ thick $\mathrm{In}$. The $\mathrm{SiN}_{\mathbf{x}}$ layer in this image is approximately $30 \mathrm{~nm}$ thick. Part (b) shows an AFM image of a single polyethylene crystal. This crystal is $\sim 10 \mathrm{~nm}$ thick. Part (c) shows an RBS spectrum of $0.4 \mathrm{~nm} \mathrm{Bi}$ on a sensor with an $\mathrm{Ag}$ heater and $\mathrm{Cr}$ adhesion layer. Peaks from the $\mathrm{Si}$ and $\mathrm{N}$ in the $\mathrm{SiN}_{\mathrm{x}}$ membrane and $\mathrm{C}$ from the graphite mask can also be seen.

heater more reliable due to a decrease in the amount of light transmitted through the metal layer.

A $50 \mathrm{~nm}$ thick metallization is found to be a good trade-off between these factors.

\section{Planar Dimensions}

Changing the planar dimensions of the calorimetric cell $(l$ and $w)$ does not change the addenda, if the total area is held constant. However, as can be seen from (10) and (12), SNR does depend on $l$ and $w$. SNR is proportional to $l$, when the heater resistance is low enough to make thermal noise negligible [see (10)]. For high $l$, this dependence is weaker (12). Heater width $w$ does

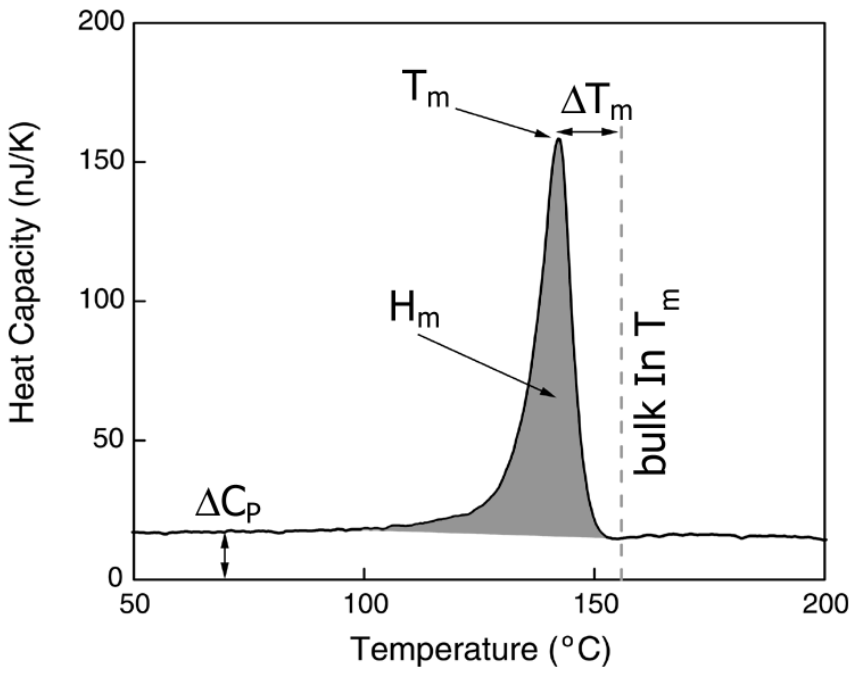

Fig. 6. Heat capacity curve of $3 \mathrm{~nm}$ In on a calorimetric sensor. Parameters of particular interest are the heat capacity of the solid phase $\Delta C_{p}$, the latent heat of fusion $H_{m}$, and the melting temperature $T_{m}$. Because of the small size of the particles, the melting temperature is smaller than the bulk melting temperature of In, $157^{\circ} \mathrm{C}$. The depression in melting point due to this size effect is also shown, as $\Delta T_{m}$.

not affect the SNR (10) until it becomes low enough to make the thermal noise dominant. Decreasing the $l / w$ ratio, or heater resistance, will have the advantage of a smaller shunting effect and the disadvantage of larger transients, as it was described for increasing of $h^{\text {Met }}$ and decreasing of $\lambda$.

Since increasing the length of heater is an effective way to enhance performance, it is useful to estimate how long the heater can be fabricated. If the heater has a shape of a straight strip, the heater length is determined by the membrane size, which is limited primarily by the strength of the $\mathrm{SiN}_{\mathrm{x}}$. The largest membranes that we have reliably fabricated were $\sim 4 \mathrm{~mm} \times 11 \mathrm{~mm}$ (and $80 \mathrm{~nm}$ thick), but our typical size is $\sim 7 \mathrm{~mm} \times 3 \mathrm{~mm}$ (and $50 \mathrm{~nm}$ thick). Serpentine-shaped heaters can lead to strong lateral nonuniformities in the temperature field in the sensing area of the device. In this case, thick pads of heat conductive material are used to eliminate temperature gradients [16], which substantially increases the addenda and complicates the sensor fabrication.

The maximum sensor size is different from the maximum membrane size. Enlarging the Si frame (and keeping the size of the membrane constant) has the advantage of strengthening the sensor, makes handling more convenient, allows more space for contact pads, increases the heat sink and improves the thermal contact with the sensor support. Increased cost and fewer sensors per wafer are disadvantages.

In the fabrication process, the dimensions of the sensor are limited by the maximum die size of the stepper used for photolithography. The $5 \times$ magnification, combined with the maximum photomask size, limits the total size of the sensor to about $15 \mathrm{~mm} \times 15 \mathrm{~mm}$.

Heat loss from the calorimetric cell is also important in design considerations of the heater geometry. During the scan, the Si frame acts as a large heat sink, because it has a much larger heat capacity than the membrane part of the sensor. The heat supplied to the sensor is also quite small, on the order of 
TABLE II

Bulk Properties of Some SAMPle Materials at 298 K

\begin{tabular}{lccccc}
\hline \hline & $\begin{array}{c}\rho \\
\left(\mathrm{g} \cdot \mathrm{cm}^{-3}\right)\end{array}$ & $\begin{array}{c}C_{P} \\
\left(\mathrm{~J} \cdot \mathrm{g}^{-1} \cdot \mathrm{K}^{-1}\right)\end{array}$ & $\begin{array}{c}T_{m} \text { or } T_{g} \\
(\mathrm{~K})\end{array}$ & $\begin{array}{c}H_{m} \\
(\mathrm{~J} / \mathrm{g})\end{array}$ & Ref. \\
\hline $\mathrm{Bi}$ & 9.79 & 0.122 & $44.6\left(T_{m}\right)$ & 53.3 & {$[32]$} \\
$\mathrm{In}$ & 7.31 & 0.233 & $429.8\left(T_{m}\right)$ & 28.6 & {$[32]$} \\
$\mathrm{Sn}$ & 7.26 & 0.228 & $505.1\left(T_{m}\right)$ & 60.4 & {$[32]$} \\
$\begin{array}{l}\text { polyethylene, } \\
\text { crystalline }\end{array}$ & 0.96 & 1.7 & $404\left(T_{m}\right)$ & 140 & {$[21]$} \\
$\begin{array}{l}\text { polystyrene, } \\
\text { amorphous }\end{array}$ & 1.04 & 1.22 & $373\left(T_{g}\right)$ & $\ldots$ & {$[33],[34]$} \\
\hline \hline
\end{tabular}

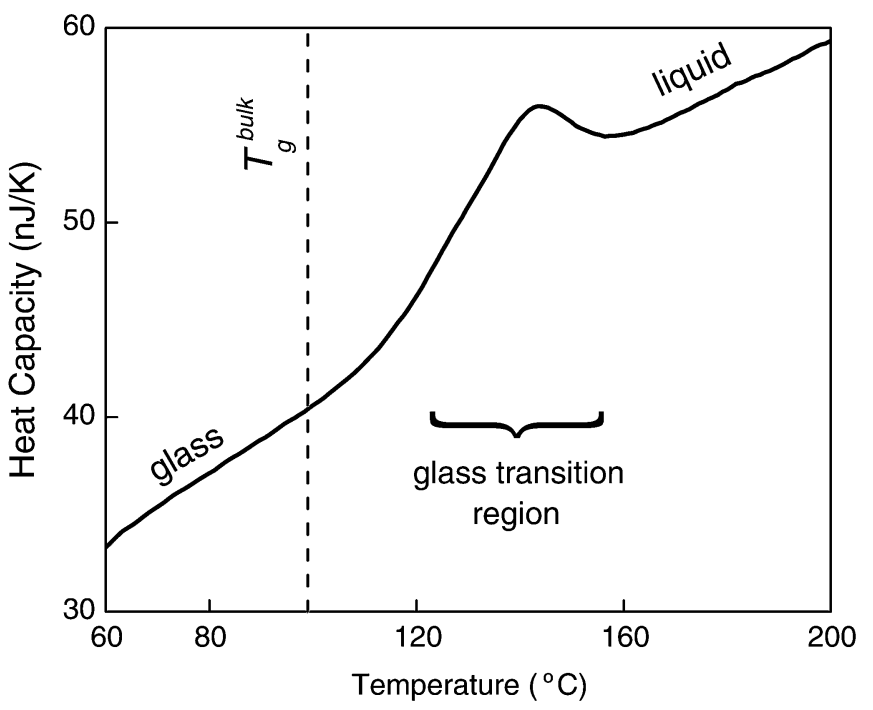

Fig. 7. Heat capacity data from a polystyrene film $7 \mathrm{~nm}$ thick. The glassy, liquid, and glass transition regions are all shown, as well as the bulk glass

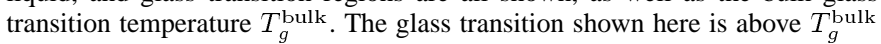
due to the high rate of cooling inherent to the nanocalorimetric technique.

several hundreds of microjoules. These two factors combine to ensure that the temperature of the heater where it overlaps the frame will be very close to the ambient even though the center of the heater may be hundreds of kelvins above ambient. Since this device is designed to be an adiabatic-type calorimeter, the heat loss can seriously effect performance. For this reason, the voltage sensing probes are located $\sim 1 \mathrm{~mm}$ away from the edge of the membrane, where the temperature is approximately uniform. Thus, we only sense the "hot" segment of the heater. There is a drawback in moving the sensing probes too close to each other, however, as it reduces $l$.

The width of the heater is also important. For a wider heater, a smaller fraction of the total heat will be lost via conduction through the $\mathrm{SiN}_{\mathrm{x}}$ membrane. If the width is too small, it is difficult to make and position the shadow mask used to locally deposit the sample during vapor deposition. Our current sensor design uses a heater $0.5 \mathrm{~mm}$ in width.

\section{APPLICATIONS}

\section{A. Sample Deposition}

The material to be measured is deposited on the sensor using various methods. Materials suitable for vacuum deposition (e.g. $\mathrm{Al}, \mathrm{Au}, \mathrm{Bi}, \mathrm{In}, \mathrm{Si}, \mathrm{Sn}$ ) are thermally evaporated onto the mem-

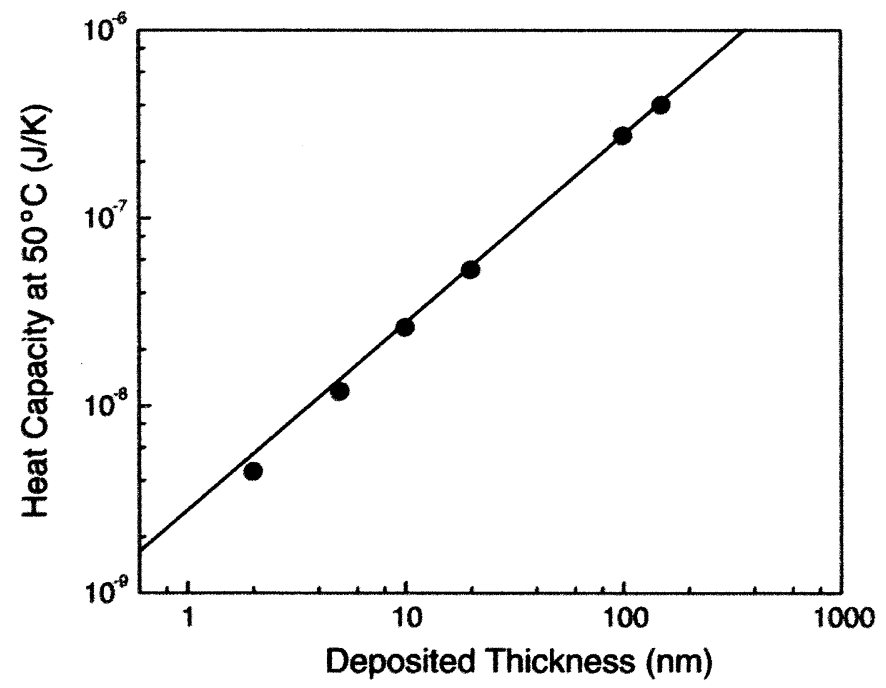

Fig. 8. Heat capacity data from a series of six Bi films of varying thickness. The heat capacity at one point, $50{ }^{\circ} \mathrm{C}$, was measured, and is shown versus the deposited thickness as measured by a quartz thin film monitor. The solid line was calculated using the $\mathrm{Bi}$ bulk specific heat at $50{ }^{\circ} \mathrm{C}$ of $0.13 \mathrm{~J} \cdot \mathrm{g}^{-1} \cdot \mathrm{K}^{-1}$, and the surface area of the shadow mask of $2.5 \mathrm{~mm}^{2}$.

brane through a metal shadow mask. All these examples are conductive (at least at the high limit of the working temperature, as in $\mathrm{Si}$ ) and must be deposited onto the $\mathrm{SiN}_{\mathrm{x}}$ side of the calorimetric cell. The mask is used to limit the area of deposition to the same, or a slightly smaller, area as the heater. In the case of $\mathrm{Bi}$ deposition, material can deposit lightly on the metallization side of the sensor (which faces away from the evaporation source) and degrades the performance of the device. Good shielding of the metal sides of both sample and reference cells is necessary to eliminate this effect. Deposition and in situ calorimetric measurements are done in vacuum of $\sim 10^{-6} \mathrm{~Pa}$.

Non-conductive material can be deposited on either side (metal or $\mathrm{SiN}_{\mathrm{x}}$ ) of the cell depending on the complexity of the tooling involved, and interaction between material and metallization or $\mathrm{SiN}_{\mathrm{x}}$. Glassy polymer films such as polystyrene, poly(2-vinyl pyridine) and poly (methyl methacrylate) are spin-cast from solution onto the metallization side of the cell [22]. The last condition is important, because these polymers do not wet the $\mathrm{SiN}_{\mathrm{x}}$ surface. It should be noted, that typical sensors are rigid enough to survive in spinning procedure, but vacuum chucks should not be used. Polymer crystals such as polyethylene are first grown in a solvent, then the suspension sprayed onto the sensor with an artist's airbrush. Self-assembled monolayers of alkanethiols are grown from alcohol solutions on $\mathrm{Au}$ layers deposited on the $\mathrm{SiN}_{\mathrm{x}}$ side of the cell. 
Even in a vacuum of $10^{-6} \mathrm{~Pa}$, if the sensor is allowed to sit for a long period of time (e.g., $1 \mathrm{~min}$ ), residual gas can adhere to the surface. During the first few heating pulses, this gas will desorb, and the energy involved in the desorption process will interfere with the signal of interest. We estimate one monolayer of adsorbed gas could draw away as much as $500 \mathrm{~nJ}$ as it desorbs from the surface.

\section{B. Sample Microanalysis}

Various microanalysis techniques can be combined with our calorimetric measurements. Some examples are shown in Fig. 5. Contact mode atomic force microscopy has been used to measure the roughness and dimensions of polymer crystals directly on the working part of the heater without rupturing the membrane [21]. Focused ellipsometry [22] has been used to measure the thickness of spin-cast polymer films on a Pt heater. We have also performed RBS [27] on sensors both before and after calorimetry to determine the amount of deposited sample. In this case, a graphite mask is used to limit the area being measured to the membrane area of the calorimetric sensor, and not the $\mathrm{Si}$ frame. We use the peak from the heater (a known area and thickness) to calibrate the sample peak.

The ability to produce good TEM images directly on the cell strongly depends on the sensor design, as it was discussed in Section V. If the sensor is not optimized for TEM, images can still be made on the sample on the $\mathrm{SiN}_{\mathrm{x}}$ very close to the heater edge. In case of vacuum deposition, the shadow mask has a $400 \mu \mathrm{m} \times 400 \mu \mathrm{m}$ notch cut into it, so a small amount of material can be deposited on the membrane next to the heater strip.

\section{Results of Selected Calorimetric Measurements}

Fig. 6 shows a heat capacity curve from an In film with an average thickness of $3 \mathrm{~nm}$, made by deposition from vapor phase onto the cold $\mathrm{SiN}_{\mathrm{x}}$ surface of calorimetric cell. A number of interesting parameters can be extracted from this curve, among them: the heat capacity of the solid phase $\Delta C_{P}$; the latent heat of fusion $H_{m}$; and the melting temperature $T_{m}$. Bulk properties for some materials under study are listed in Table II.

The mass of the deposited material can be calculated from $C_{P}$, using the specific heat capacity $c_{P}$ for bulk material. This method is accurate as long as $c_{P}$ is not susceptible to size and interface effects.

In traditional DSC measurements of bulk metal, the caloric curve would show a very sharp spike at bulk melting temperature $T_{m}^{\text {bulk }}$. Fig. 5(a) shows a TEM micrograph of a sample film, a $3 \mathrm{~nm}$ In deposited onto a calorimeter. As can be seen in the image, very thin films such as this one consist of isolated particles. Size effects have lowered the $T_{m}$ and $H_{m}$ for the particles [7]. The width of the peak in Fig. 6 is due to the distribution of In particle sizes.

In addition to the size effects, the high scanning rates intrinsic to adiabatic nanocalorimetry can also affect the calorimetric behavior of material. The glass transition in a $7 \mathrm{~nm}$ thick polystyrene film (with molecular weight characteristics $M_{w}=$ $120200, M_{w} / M_{n}=1.04$ ) is demonstrated in Fig. 7. Due to a cooling rate of $\sim 3 \mathrm{~K} / \mathrm{ms}$, the glass transition region is well above of the bulk value of $99^{\circ} \mathrm{C}$. The bulk value was measured for this sample using a heating rate of $3 \mathrm{~K} / \mathrm{min}$, common for conventional DSC devices.

A detailed treatment of the sensitivity and accuracy specifications of the nanocalorimeter is well beyond the scope of this paper. For this work, however, we demonstrate the efficiency of the system by a few examples.

One practical approach in evaluating performance is by measuring the response of the nanocalorimeter to the addition of known amounts of material. Fig. 8 shows the heat capacity measured at $50{ }^{\circ} \mathrm{C}$ for six Bi films with different thicknesses. The thickness shown was measured by a crystal monitor, used as a standard reference. Over the given range, the measured $C_{P}$ increases linearly with film thickness and agrees well with values, calculated from crystal monitor data and bulk $c_{P}$, to within $20 \%$.

To characterize performance on an even more sensitive scale, we measured the incremental change in the heat capacity $C_{P}$ during slow $(<0.1 \mathrm{~nm} / \mathrm{min})$ continuous deposition of indium. The $C_{P}$ monotonically increases with In thickness, with a resolution of $4 \times 10^{-3} \mathrm{~nm}$ in terms of thickness and $30 \mathrm{pJ} / \mathrm{K}$ in terms of heat capacity [28].

In summary, we have discussed the fabrication and operation of a MEMS, thin-film, differential scanning calorimeter and have evaluated the performance of system under typical operating conditions.

\section{ACKNOWLEDGMENT}

The authors are particularly indebted to P. Infante at the Cornell Nanofabrication Facility for his assistance and expertise in the sensor fabrication. The AFM image in Fig. 5 was taken by A. Kwan. Microanalysis was carried out in the Center for Microanalysis of Materials, University of Illinois, which is partially supported by the U.S. Department of Energy under Grant DEFG02-91-ER45439. They also thank D. Cahill for his valuable insight and discussions, and J. Mayer for his helpful suggestions.

\section{REFERENCES}

[1] D. Golmayo and J. L. Sacedon, "Measurements and interpretation of the electrical resistivity and Hall coefficient in polycrystalline gold films. I," Thin Solid Films, vol. 35, pp. 137-141, 1976.

[2] O. Akcakir, J. Therrien, G. Belomoin, N. Barry, J. D. Muller, E. Gratton, and M. Nayfeh, "Detection of luminescent single ultrasmall silicon nanoparticles using fluctuation correlation spectroscopy," Appl. Phys. Lett., vol. 76, pp. 1857-1859, 2000.

[3] M. G. Mitch, S. J. Chase, J. Fortner, R. Q. Yu, and J. S. Lannin, "Phase transition in ultrathin Bi films," Phys. Rev. Lett., vol. 67, p. 875, 1991.

[4] J. P. K. Doye and F. Calvo, "Entropic effects on the size dependence of cluster structure," Phys. Rev. Lett., vol. 86, pp. 3570-3573, 2001.

[5] G. L. Allen, R. A. Bayles, W. W. Gile, and W. A. Jesser, "Small particle melting of pure metals," Thin Solid Films, vol. 144, pp. 297-308, 1986.

[6] P. Buffat and J. P. Borel, "Size effect on the melting temperature of gold particles," Phys. Rev. A, vol. 13, pp. 2287-2298, 1976.

[7] M. Zhang, M. Y. Efremov, F. Schiettekatte, E. A. Olson, A. T. Kwan, S. L. Lai, T. Wisleder, J. E. Greene, and L. H. Allen, "Size-dependent melting point depression of nanostructures: nanocalorimetric measurements," Phys. Rev. B, vol. 62, pp. 10 548-10 557, 2000.

[8] M. Y. Efremov, F. Schiettekatte, M. Zhang, E. A. Olson, A. T. Kwan, R. S. Berry, and L. H. Allen, "Discrete periodic melting point observations for nanostructure ensembles," Phys. Rev. Lett., vol. 85, pp. 3560-3563, 2000.

[9] T. P. Martin, T. Bergmann, H. Gohlich, and T. Lange, "Observation of electronic shells and shells of atoms in large Na clusters," Chem. Phys. Lett, vol. 172, pp. 209-213, 1990. 
[10] M. Schmidt, R. Kusche, B. von Issendorff, and H. Haberland, "Irregular variations in the melting point of size-selected atomic clusters," Nature, vol. 393, pp. 238-240, 1998.

[11] S. C. Wang and G. Ehrlich, "Equilibrium shapes and energetics of iridium clusters on $\operatorname{Ir}(111)$, , Surf. Sci., vol. 391, pp. 89-100, 1997.

[12] K. D. Irwin, G. C. Hilton, D. A. Wollman, and J. M. Martinis, "X-ray detection using a superconducting transition-edge sensor microcalorimeter with electrothermal feedback," Appl. Phys. Lett., vol. 69, pp. 1945-1947, 1996

[13] D. McCammon, S. H. Moseley, J. C. Mather, and R. F. Mushotzky, "Experimental tests of a single-photon calorimeter for X-ray spectroscopy," J. Appl. Phys., vol. 56, pp. 1263-1266, 1984.

[14] J. S. Suehle, R. E. Cavicchi, M. Gaitan, and S. Semancik, "Tin oxide gas sensor fabricated using CMOS micro-hotplates and in-situ processing," IEEE Electron Device Lett., vol. 14, pp. 118-120, 1993.

[15] F. Nuscheler, "The microcalorimeter, a silicon gas sensor," Arch. Elektronik Übertragungstechnik, vol. 42, pp. 80-84, 1988.

[16] D. W. Denlinger, E. N. Abarra, K. Allen, P. W. Rooney, M. T. Messer, S. K. Watson, and F. Hellman, "Thin film microcalorimeter for heat capacity measurements from 1.5 to $800 \mathrm{~K}$," Rev. Sci. Instrum., vol. 65, pp. 946-959, 1994.

[17] Y. Nakagawa, R. Schafer, and H. J. Guntherodt, "Picojoule and submillisecond calorimetry with micromechanical probes," Appl. Phys. Lett., vol. 73, pp. 2296-2298, 1998.

[18] M. Mitome, "In-situ observation of melting of fine lead particles by high-resolution electron microscopy," Surf. Sci., vol. 442, pp. L953-L958, 1999.

[19] S. L. Lai, G. Ramanath, L. H. Allen, P. Infante, and Z. Ma, "High-speed $\left(10^{\wedge} 4\right.$ degrees $\left.\mathrm{C} / \mathrm{s}\right)$ scanning microcalorimetry with monolayer sensitivity (J/m^2)," Appl. Phys. Lett., vol. 67, pp. 1229-1231, 1995.

[20] E. A. Olson, M. Y. Efremov, A. T. Kwan, S. Lai, V. Petrova, F. Schiettekatte, J. T. Warren, M. Zhang, and L. H. Allen, "Scanning calorimeter for nanoliter-scale liquid samples," Appl. Phys. Lett., vol. 77, pp. 2671-2673, 2000.

[21] A. T. Kwan, M. Efremov, E. A. Olson, F. Schiettekatte, M. Zhang, P. H. Geil, and L. H. Allen, "Nanoscale calorimetry of isolated polyethylene single crystals," J. Polym. Sci. B Polym. Phys., vol. 39, pp. 1237-1245, 2001.

[22] M. Y. Efremov, J. T. Warren, E. A. Olson, M. Zhang, A. T. Kwan, and L. H. Allen, "Thin-film differential scanning calorimetry: a new probe for assignment of the glass transition of ultrathin polymer films," Macromolecules, vol. 35, pp. 1481-1483, 2002.

[23] G. T. A. Kovacs, Micromachined Transducers Sourcebook. Burr Ridge, IL: WCB/McGraw-Hill, 1998.

[24] G. Wedler and G. Alshorachi, "The influence of thickness on the resistivity, the temperature coefficient of resistivity and the thermoelectric power of evaporated palladium films at $77 \mathrm{~K}$ and $273 \mathrm{~K}$," Thin Solid Films, vol. 74, pp. 1-16, 1980.

[25] P. E. Laibinis, J. J. Hickman, M. S. Wrighton, and G. M. Whitesides, "Orthogonal self-assembled monolayers: alkanethiols on gold and alkane carboxylic acids on alumina," Science, vol. 245, pp. 845-847, 1989.
[26] J. A. Mayer and S. S. Lau, Electronic Materials Science: For Integrated Circuits in Si and GaAs. New York: Macmillan, 1990.

[27] L. C. Feldman and J. W. Mayer, Fundamentals of Surface and Thin Film Analysis. New York: North-Holland, 1986.

[28] M. Zhang, M. Y. Efremov, E. A. Olson, S. Zhang, and L. H. Allen, "Real-time heat capacity measurement during thin-film deposition by scanning nanocalorimetry," Appl. Phys. Lett., vol. 81, pp. 3801-3803, 2002.

[29] D. R. Lide, CRC Handbook of Chemistry and Physics. New York: CRC Press, 2002, vol. 82.

[30] G. V. Samsonov, Handbook of the Physicochemical Properties of the Elements. New York: IFI/Plenum, 1968.

[31] C. H. Mastrangelo, T. Yu-Chong, and R. S. Muller, "Thermophysical properties of low-residual stress, silicon-rich, LPCVD silicon nitride films," Sens. Actuators: Phys. A, vol. A23, pp. 856-880, 1990.

[32] D. R. Lide, CRC Handbook of Chemistry and Physics, 82 ed. Boca Raton, FL: CRC, 2001

[33] Y. Yang, "Thermal conductivity," in Physical Properties of Polymers Handbook, J. E. Mark, Ed. Woodbury, NY: AIP, 1996, pp. 111-117.

[34] J. Wen, "Heat capacities of polymers," in Physical Properties of Polymers Handbook, J. E. Mark, Ed. Woodbury, NY: AIP, 1996, pp. 101-109.

[35] S. N. Kaul, "Low-temperature magnetization and spin-wave excitations in amorphous Ni-rich transition-metal-metalloid alloys," Phys. Rev. B, vol. 27, pp. 5761-5774, 1983.

Eric A. Olson, photograph and biography not available at the time of publication.

Mikhail Yu. Efremov, photograph and biography not available at the time of publication.

Ming Zhang, photograph and biography not available at the time of publication.

Zishu Zhang, photograph and biography not available at the time of publication.

Leslie H. Allen, photograph and biography not available at the time of publication. 